\title{
Tactile Spatial Attention Enhances Gamma-Band Activity in Somatosensory Cortex and Reduces Low-Frequency Activity in Parieto-Occipital Areas
}

\author{
Markus Bauer, ${ }^{1,2}$ Robert Oostenveld, ${ }^{1,3}$ Maarten Peeters, ${ }^{1}$ and Pascal Fries ${ }^{1,2}$ \\ ${ }^{1}$ F. C. Donders Centre for Cognitive Neuroimaging, Radboud University Nijmegen, 6525 EN Nijmegen, The Netherlands, ${ }^{2}$ Department of Biophysics, \\ Radboud University Nijmegen, 6525 EZ Nijmegen, The Netherlands, and ${ }^{3}$ Center for Sensori-Motor Integration, University of Aalborg, 9220 Aalborg, \\ Denmark
}

We investigated the effects of spatial-selective attention on oscillatory neuronal dynamics in a tactile delayed-match-to-sample task. Whole-head magnetoencephalography was recorded in healthy subjects while dot patterns were presented to their index fingers using Braille stimulators. The subjects' task was to report the reoccurrence of an initially presented sample pattern in a series of up to eight test stimuli that were presented unpredictably to their right or left index finger. Attention was cued to one side (finger) at the beginning of each trial, and subjects performed the task at the attended side, ignoring the unattended side.

After stimulation, high-frequency gamma-band activity $(60-95 \mathrm{~Hz})$ in presumed primary somatosensory cortex (S1) was enhanced, whereas alpha- and beta-band activity were suppressed in somatosensory and occipital areas and then rebounded. Interestingly, despite the absence of any visual stimulation, we also found time-locked activation of medial occipital, presumably visual, cortex. Most relevant, spatial tactile attention enhanced stimulus-induced gamma-band activity in brain regions consistent with contralateral S1 and deepened and prolonged the stimulus induced suppression of beta- and alpha-band activity, maximal in parieto-occipital cortex. Additionally, the beta rebound over contralateral sensorimotor areas was suppressed.

We hypothesize that spatial-selective attention enhances the saliency of sensory representations by synchronizing neuronal responses in early somatosensory cortex and thereby enhancing their impact on downstream areas and facilitating interareal processing. Furthermore, processing of tactile patterns also seems to recruit visual cortex and this even more so for attended compared with unattended stimuli.

Key words: synchronization; oscillation; gamma; attention; somatosensory; MEG; Braille

\section{Introduction}

Early studies investigating the mechanisms of spatial-selective attention in humans have used event-related potentials and have found increased sensory responses to attended compared with ignored stimuli (Groves and Eason, 1969; Hillyard et al., 1973). These effects have been corroborated in numerous experiments using both EEG/magnetoencephalography (MEG) and functional magnetic resonance imaging (MRI)/positron emission tomography (Brefczynski and DeYoe, 1999; Mangun et al., 2001) and have led to the conclusion that attention enhances the strength of the neuronal representations of attended stimuli, such that they have improved access to higher processing resources. Experiments using single-cell recordings in monkeys have re-

Received Dec. 22, 2004; revised Nov. 16, 2005; accepted Nov. 17, 2005.

This research was supported by The Netherlands Organization for Scientific Research Grants 452-03-344 and 051-02-050 (P.F.), Human Frontier Science Program Organization Grant RGP0070/2003 (P.F.), Volkswagen Foundation Grant I/79876 (P.F.), and Danish Technical Research Council Grant 26-01-0092 (R.0.). We thank Hubert Preissl and Jürgen Dax for valuable information about the Braille stimulators and Erik van den Boogert for help in building the devices. Furthermore, we thank Ole Jensen for helpful discussions.

Correspondence should be addressed to Markus Bauer, F. C. Donders Centre for Cognitive Neuroimaging, Adelbertusplein 1, 6525 EK Nijmegen, The Netherlands. E-mail: markus.bauer@fcdonders.ru.nl.

D01:10.1523/JNEUROSCI.5228-04.2006

Copyright $\odot 2006$ Society for Neuroscience $\quad$ 0270-6474/06/260490-12\$15.00/0 vealed that multiple simultaneously presented stimuli compete for access to higher-level processing and that this competition is biased in favor of the attended stimulus. More specifically, Reynolds et al. (1999) presented two stimuli within the receptive field of a neuron in monkey area V4. When attention was directed to one of the stimuli, the neuron responded as if there were only the attended stimulus. What mechanisms increase the efficacy of an attended stimulus compared with an unattended stimulus?

It has been proposed recently that this modulation of input efficacy might be accomplished by a regulation of the synchronization among the involved neurons (Engel et al., 2001; Fries et al., 2001a,b; Salinas and Sejnowski, 2001). Synchronous input to a postsynaptic target neuron is known to have a greater impact than asynchronous input. A recent study in awake trained monkeys has indeed shown enhanced synchrony of stimulus-induced gamma-band oscillations in response to attended compared with unattended stimuli (Fries et al., 2001b). This is consistent with the finding of enhanced gamma-band activity for attended stimuli over parieto-occipital electrodes in the human EEG (Gruber et al., 1999). Furthermore, it has been shown that stimulus selection in binocular rivalry is associated with enhanced gamma-band synchrony in primary visual cortex (Fries et al., 1997, 2002).

Much less is known about how spatial-selective attention acts 

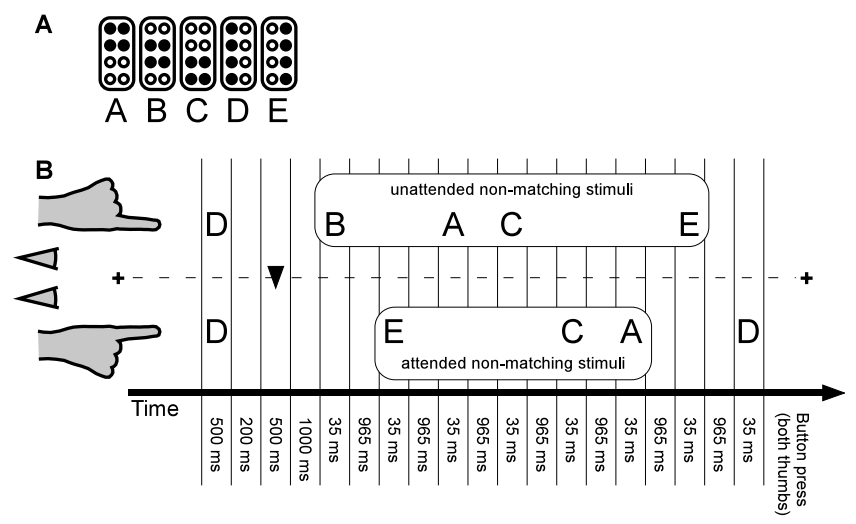

Figure 1. The spatial-selective delayed-match-to-sample task. $A$, The Braille patterns used. $B$, An example sequence is shown with the timing of sample, cue, and test presentations and of the response. Sequences could have any length between one and eight test stimuli.

on individual neurons in the somatosensory system. One consistent finding from both animal (Hsiao et al., 1993; Burton et al., 1997; Burton and Sinclair, 2000a; Meftah et al., 2002) and human studies is that attentional effects are more robustly found in secondary somatosensory cortex (S2) and only to a much lesser degree in primary somatosensory cortex (S1) (Burton and Sinclair, 2000b; Hoechstetter et al., 2000). Furthermore, Steinmetz et al. (2000) recently found increased synchrony of neural responses in S2 when a monkey had to attend to tactile stimuli compared with when he had to attend to visual stimuli. However, it is not clear how results from this intermodal attention task relate to spatial-selective tactile attention. Also, this study did not analyze synchronization as a function of frequency, and therefore it is not clear how the enhanced synchronization described there relates to the enhanced gamma-band synchronization found with spatial-selective visual attention (Fries et al., 2001b).

Here, we investigated the effects of tactile attention on oscillatory neuronal synchrony and more specifically address the question whether spatial-selective tactile attention enhances gamma-band activity in the human somatosensory system. Therefore, we recorded MEG in healthy human subjects and used mechanical Braille stimuli in a tactile delayed-match-to-sample task.

\section{Materials and Methods}

Subjects. Subjects were nine adult volunteers (four males, five females; mean \pm SD age, $25.1 \pm 2.7$ years) who were recruited primarily from the Radboud University Nijmegen. All subjects provided written consent according to institutional guidelines of the local ethics committee (Commissie Mensgebonden Onderzoek Region Arnhem-Nijmegen, The Netherlands), were reported to be free of mental and neurological illness, and were right-handed as assessed by the Edinburgh Handedness Inventory (Oldfield, 1971).

Stimuli. Tactile stimuli were presented to the left or right index fingers by means of two piezoelectrical Braille stimulators (Metec, Stuttgart, Germany) that were driven using custom-built electronic circuitry. Each Braille stimulator had a matrix of two columns by four rows of individually controllable pins, which could be raised and lowered with a 1-ms precision. The stimulus set consisted of five different dot patterns that were created with the constraint that, for each pattern, four pins were raised in clusters of at least two adjacent pins (in either horizontal or vertical direction; for details, see Fig. $1 A$ ). Stimulation consisted of elevating the pin pattern by $\sim 2 \mathrm{~mm}$, holding them elevated for $500 \mathrm{~ms}$ (sample stimulus) or $35 \mathrm{~ms}$ (test stimuli), and then lowering them again. While in the elevated position, the pins were stationary. The low currents necessary for driving piezoelectrical membranes created only small and short-lived artifacts. Those artifacts occurred at two times: (1) during the specification of the stimulus pattern in the electronic circuit driving the Braille cells, which happened $100 \mathrm{~ms}$ before actual stimulus delivery; and (2) during the actual delivery of the stimulus. These short ( $\sim 1 \mathrm{~ms})$ artifacts can be seen in the time-domain averages in Figure 2. No artifacts were measured when the stimulus remained stationary (pins either elevated or lowered).

Task. The task was a spatial-selective tactile delayed-match-to-sample task that subjects performed while continuously fixating a central fixation cross. The temporal structure of the task is outlined in Figure $1 B$. Each trial started with the presentation of the tactile sample stimulus (selected randomly from the stimulus set for each trial) to both fingers for $500 \mathrm{~ms}$. Two hundred milliseconds after sample offset, a visual arrow was foveally presented for $500 \mathrm{~ms}$ and cued spatial-selective attention to either the right or left index finger. After the offset of the cue, there was no change in visual stimulation anymore (only stationary fixation cross). One second after the offset of the visual cue, the presentation of a series of up to eight test stimuli was started, and these stimuli were delivered unpredictably to either the right or the left index finger. The test stimuli were chosen randomly out of the set of five dot patterns (see above) and were presented each for $35 \mathrm{~ms}$, and stimulus onsets were separated by $1000 \mathrm{~ms}$. Short presentation time was chosen for several reasons: (1) to prevent subjects from making exploratory finger movements that might have confounded the attentional effects; (2) to avoid a separate off response during stimulus processing; and (3) to increase task difficulty and force subjects to really attend to the precued finger. The subjects' task was to press a button lying between both hands with both thumbs as soon as they recognized the occurrence of a target (i.e., the sample pattern) on the precued (attended) side. The button was constructed such that it only counted the response when pressed with both hands. No button should be pressed when a sample pattern was presented on the uncued (unattended) side. The trial stopped after presentation of a target, after the subject had pressed the button erroneously, or after eight stimuli had been presented. In $\sim 25 \%$ of the trials, no target was presented. Subjects were given feedback about the correctness of their behavior at the end of each trial. We restricted our analysis to correctly rejected nonsample patterns and therefore stimuli to which no motor response was given.

Procedure. After subjects were seated comfortably, electrodes and localization coils were attached (see below). Before the recording session, each pattern was presented 10 times in pseudorandom order to both the left and right index finger to familiarize the subject with the stimuli. A set of two experimental blocks with 16 trials each was given for training purposes. Throughout the recording session, auditory white noise was presented to the subjects through pneumatic earphones to mask the sound generated by the Braille cells.

The recording session consisted of 16 blocks of 16 trials each, resulting in $\sim 1 \mathrm{~h}$ of recording time. After the recording session, structural MRIs of each individual subject were made on a $1.5 \mathrm{~T}$ Siemens Sonata scanner (Siemens, Erlangen, Germany) using a standard T1-weighted sequence to enable reconstruction of each subjects head shape for the later described interpolation and source reconstruction procedures.

$M E G$ recordings. Neuromagnetic activity was recorded using the whole-head 151 channel axial-gradiometer MEG system (Omega 2000; CTF Systems, Port Coquitlam, British Columbia, Canada) at the F. C. Donders Centre for Cognitive Neuroimaging. In addition, the electrocardiogram and vertical and horizontal electrooculograms (EOGs) were recorded through appropriate bipolar montages. The subject's head position relative to the MEG sensors was measured at the beginning and at the end of the session using three magnetic coils. One such coil was placed on the nasion. The other two coils were mounted onto the earpieces that held the tubes for pneumatic auditory stimulation such that the tubes ran through the middle of the magnetic coils. During the structural MRI scans, we used the same ear pieces but then with short tubes that contained a drop of vitamin $\mathrm{E}$ in place of the coils. This procedure allowed precise and reliable coregistration of the MEG and the MRI data. MEG data were low-pass filtered at $300 \mathrm{~Hz}$ and sampled continuously at a rate of $1200 \mathrm{~Hz}$.

Data analysis. Data were analyzed using the Fieldtrip software package (http://www.ru.nl/fcdonders/fieldtrip/), a Matlab-based toolbox for the 
analysis of electrophysiological data that has been developed by our group. Data were checked for artifacts using a semiautomatic routine that helped detecting and rejecting eye blinks, muscle artifacts, and jumps in the MEG signal caused by the SQUID electronics. For each of these artifacts, an appropriate metric with specific sensitivity for the respective artifact was computed, and a rejection threshold was determined empirically. This was done separately for each subject, but subsequently, artifact rejection parameters for a given subject were fixed and applied automatically to its entire dataset. Adjustments to individual subjects' data are necessary because of differences in noise levels and in the signalto-noise ratio of the EOG recordings.

The power-line artifact was removed using the following procedure. All signals had been recorded continuously for the entire duration of the recording session. For each time epoch of interest (and each recording channel), we first took a 10 s epoch out of the continuous signal with the epoch of interest in the middle. We then calculated the discrete Fourier transform (DFT) of the $10 \mathrm{~s}$ epoch at 50,100 , and $150 \mathrm{~Hz}$ without any tapering. Because the power-line artifact is of a perfectly constant frequency, the $10 \mathrm{~s}$ epoch contains integer cycles of the artifact frequencies, and all of the artifact energy is contained in those DFTs. We then constructed 50,100, and $150 \mathrm{~Hz}$ sine waves with the amplitudes and phases as estimated by the respective DFTs and subtracted those sine waves from the $10 \mathrm{~s}$ epoch. The epoch of interest was then cut out of the cleaned $10 \mathrm{~s}$ epoch. Power spectra of the cleaned $10 \mathrm{~s}$ epochs demonstrated that all artifact energy was eliminated, leaving a notch of a bin width of $0.1 \mathrm{~Hz}$ (equivalent to $1 / 10 \mathrm{~s}$ ). The actual spectral analysis used the multi-taper method, with a spectral smoothing of $\pm 2.5 \mathrm{~Hz}$ for the lower-frequency range and $\pm 10 \mathrm{~Hz}$ for the higher-frequency range. Thus, the notch typically became invisible.

The artifact-free data were interpolated to a common sensor array template using a minimum-norm projection method (Knösche, 2002). Subsequently, planar gradients of the MEG field distribution were calculated using a nearest-neighbor method comparable with the method described by Bastiaansen and Knösche (2000).

Spectral analysis. We performed time-resolved spectral analyses using the multi-taper method, which offers optimal spectral concentration (similar to spectral smoothing) over the frequency range of interest, i.e., it allows to trade resolution in the frequency domain for reduced variance (Mitra and Pesaran, 1999). Two frequency ranges were analyzed separately with different window lengths and spectral concentrations. The lower-frequency band ranged from 5 to $40 \mathrm{~Hz}$ and was analyzed with a window length of $400 \mathrm{~ms}$ and a spectral concentration of $\pm 2.5 \mathrm{~Hz}$. The higher-frequency band ranged from 40 to $180 \mathrm{~Hz}$ and was analyzed with a window length of $200 \mathrm{~ms}$ and a spectral concentration of $\pm 10 \mathrm{~Hz}$.

Power spectra were separately computed for the horizontal and vertical planar gradients, and the resultant vector length of both was computed to obtain the power at that sensor location regardless of the orientation of the gradient. The variance of the power in each time-frequency bin was estimated using a jackknife procedure (Efron and Tibshirani, 1993). Subsequently, $t$ statistics were calculated between all time-frequency bins and a common baseline (effective time windows, -400 to 0 $\mathrm{ms}$ for low frequencies and -250 to $0 \mathrm{~ms}$ for high frequencies). The resulting $t$ values were transformed into $z$ scores, averaged over local sensor groups, and pooled across subjects using the following formula:

$$
z=\frac{1}{N} \sum_{i=1}^{N} z_{i}
$$

with $z_{i}$ being the $z$ score of the $i$ th subject.

Those grand-average time-frequency $z$ images showed clear spectral components and allowed the definition of time-frequency windows of interest. To test the statistical significance of the effects of attention on the power in the different time-frequency windows of interest, the Fourier transform was calculated using the multi-taper method with the time windows and spectral concentrations set to the respective time-frequency window of interest. For each subject, a $z$ score was determined in the same manner as for the time-frequency analysis. To compare the two attention conditions, a paired $t$ test was performed between the $z$ scores of all subjects. The $z$ scores are well normalized for intrasubject variance and therefore can be better compared across subjects than differences in absolute power.

To examine the phase relation of the measured oscillations to the onset of the stimulus, we repeated the same time-frequency analysis on the (non-bandpass filtered) evoked fields (see description below). Additionally, we calculated the time-resolved phase-locking factor by doing a time-frequency analysis with the same settings as described above, but followed by normalizing the complex Fourier spectra on each trial by their vector length and averaging those unit-length vectors across trials (Lachaux et al., 1999).

Source analysis. For the reconstruction of the neuronal sources of the spectral components that showed a statistically significant effect of stimulation or attention, we used an adaptive spatial filtering technique (Gross et al., 2001). Each subject's brain volume was divided into a regular $8 \mathrm{~mm}$ grid, and, for each grid location, a spatial filter was constructed. This filter has the property that it passes activity from the location of interest with unit gain but suppresses all other activity. The spatial filter $w(r, f)$ is calculated according to the following formula:

$$
w(r, f)=\left(L^{\prime}(r)(C(f)+\lambda \times I)^{-1} L(r)\right)^{-1} L^{\prime}(r)(C(f)+\lambda \times I)^{-1},
$$

where $L(r)$ is the forward model (lead-field matrix) at the location $r$ of interest, $C(f)$ is the cross-spectral density (CSD) matrix between all MEG signals at the frequency $f$, and $\lambda$ is the regularization parameter. To compute the lead-field matrix, we used a multisphere model in which, for each sensor, a sphere was fitted to the head surface underneath that sensor. The head shape was derived from each individual subject's structural MRI and aligned to the MEG data.

To optimally capture the effect of interest, a time-frequency window was specified individually for each subject based on the stimulus-induced power changes. A window of the same extent in time and frequency was also placed into the baseline period. For some of the source analyses, individual subjects were excluded because it was either impossible to find a proper baseline or because the signal-to-noise ratio was not sufficient. This will be described in detail in Results. The CSD matrix was calculated directly for the entire time window of interest using multi-tapers. Noise in the CSD estimate was minimized by choosing one frequency bin and adjusting the number of tapers (and thereby integration in the frequency domain) such that the entire frequency range of interest was captured. The time windows were chosen to represent an integer number of cycles of an oscillation of the center frequency, and the spectral concentration was chosen to be an integer multiple of the Rayleigh frequency of the selected window length.

Consecutively, the power $p$ was estimated for each grid location $r$ and separately for the two conditions that were compared (stimulus effect: baseline and stimulation; attention effect: attended and nonattended) according to the following formula:

$$
p(r, f)=w(r, f) C(f)^{\prime} w^{*}(r, f) .
$$

To quantify the effects of tactile stimulation and tactile attention on oscillatory activity, we computed a voxel-wise $t$ statistic. To this end, the cross-spectral density matrices were calculated for each single trial and then jackknife averaged. The spatial filter was constructed for each individual jackknife subsample, resulting in an estimate of the mean and the variance of power at each grid location and for each condition. A $t$ statistic was then determined for the difference in power between the respective conditions. The $t$ values were subsequently $z$ transformed. To analyze the effect of stimulation, we chose, for each spectral component separately, the attention condition that provided the highest signal-tonoise ratio. Because for one comparison (effect of attention on alpha activity), the baseline levels were systematically different for the attended compared with the unattended condition, we used for this comparison not a simple $t$ test but the following contrast: (AttendedPostStim - AttendedPreStim) - (UnattendedPostStim - UnattendedPreStim).

Using SPM2 (http://www.fil.ion.ucl.ac.uk/spm), the individual anatomical MRIs and the corresponding statistical maps were spatially normalized toward the International Consortium for Brain Mapping template (Montreal Neurological Institute, Montreal, Quebec, Canada). The 


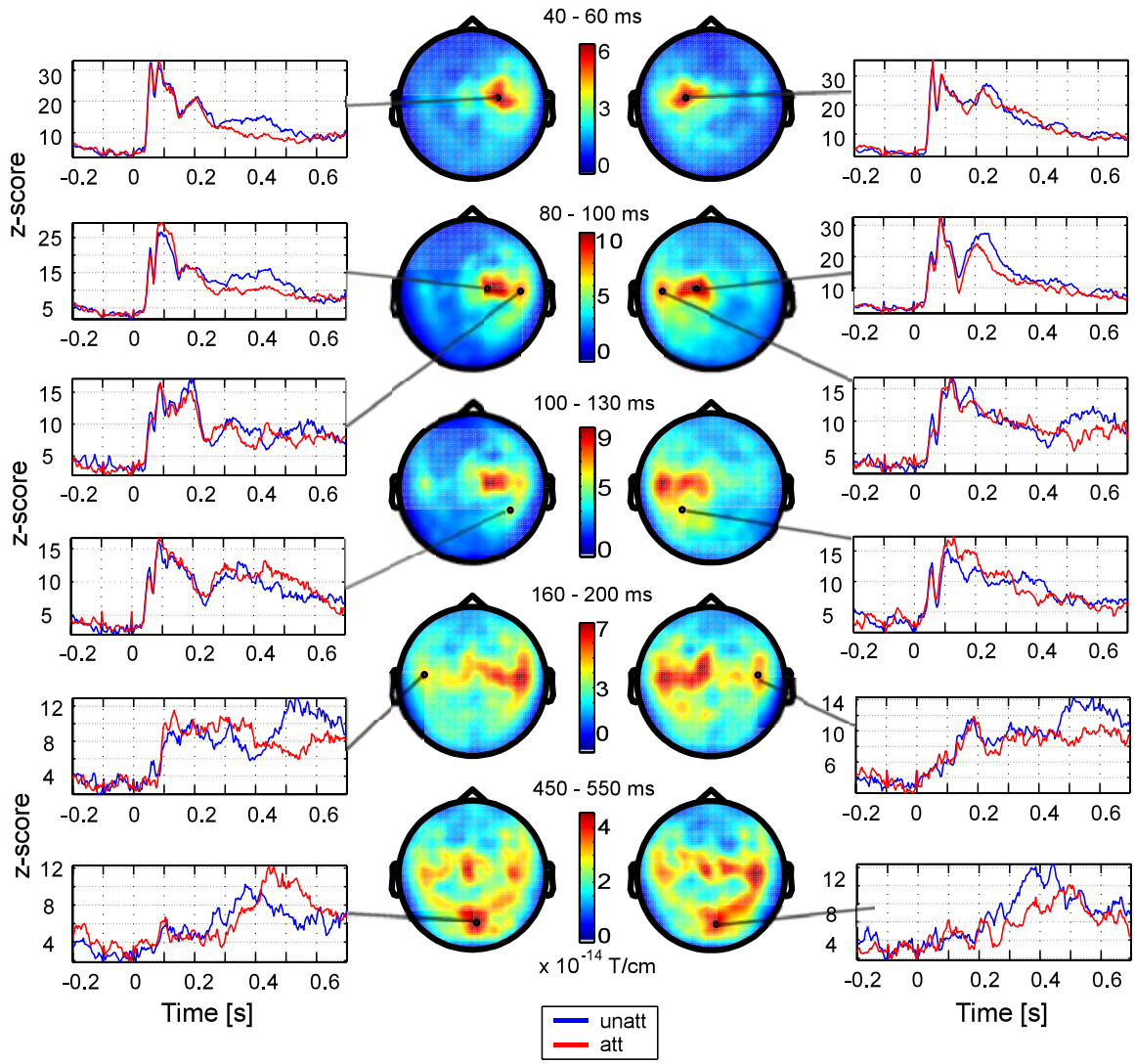

Figure 2. Planar gradients of evoked fields (for details, see Materials and Methods). Left column, Time courses of activity for left-hand stimulation in individual sensors as marked in the topographies in the middle column. Stimulus onset is at $0 \mathrm{~s}$. Blue traces are for the unattended stimuli, and red traces are for the attended stimuli. Shown are $z$ scores indicating the deviation from baseline level. Middle column, Grand-averaged topographies during epochs of interest for left- and right-hand stimulation (left and right panels, respectively). Right column, Time courses of individual sensors for stimulation of the right hand for attended (blue) and unattended (red) stimuli (z scores).

individual spatially normalized statistical maps were subsequently pooled to obtain a fixed-effect statistic using the same formula as for the sensor data.

Additionally, we performed a source reconstruction of the first two peaks of the somatosensory-evoked field to investigate the overlap of the frequency domain results with those of the well described generators of early evoked activity. To this end, we also used an adaptive spatialfiltering approach, similar to the one described above, but with the crossspectral density matrix replaced by its time-domain equivalent, the covariance matrix between all MEG signals (Van Veen et al., 1997). This was done separately for a prestimulus and a poststimulus time window, and subsequently, the same statistical and cross-subject pooling procedures were applied as for the frequency domain data.

Time-domain analysis. The same artifact-corrected raw data as used for the spectral analysis were low-pass filtered (cutoff frequency set to 40 $\mathrm{Hz}$ ), jackknife averaged, and baseline corrected (baseline from $-100 \mathrm{~ms}$ to stimulus onset), interpolated to the common sensor array and planar gradiometer transformed. The obtained realigned planar gradients were then grand averaged to obtain the average topographies. The subsequent analysis proceeded similarly to that of the spectral analysis: mean and variance were estimated based on the jackknife repetitions, and subsequently, a $t$ statistic was calculated for the difference between each sample measured after stimulus onset and the mean amplitude during the baseline period. The $t$ values were then $z$ transformed and pooled across subjects. Finally, the time courses for the attended and unattended conditions were subjected to a paired $t$ test to test for statistically significant differences between the conditions. However, because an analysis of time-locked effects was not the focus of the present work, we only tested for the presence of such effects.

\section{Results}

\section{Behavioral results}

Subjects performed the task at high accuracy levels and categorized between 77 and $94 \%$ of all stimuli correctly (mean \pm SD, $89 \pm 5.9 \%)$. Mean reaction times to the match stimuli were $505 \pm 59 \mathrm{~ms}$ for righthand stimulation and $518 \pm 63 \mathrm{~ms}$ for lefthand stimulation.

\section{Effects of tactile stimulation in the time domain}

Figure 2 shows the time courses and averaged topographies of the planar gradientevoked fields. The magnitude of the planar gradients can only take positive values and therefore relates to the absolute dipole moment independent of the dipole orientation. The planar gradient is usually maximal for sensors directly overlying the cortical sources and therefore provides a rough estimate of the underlying source distribution (Bastiaansen and Knösche, 2000). Also, compared with axial gradients, the use of planar gradients is beneficial when grand averaging across subjects, because it minimizes the risk of cancellation as a result of interindividual differences in dipole orientation. Topographies show the grand average of the planar gradients, and time courses show the pooled $z$ scores of the deviation of the signal from baseline. The two small peaks at stimulus onset and $100 \mathrm{~ms}$ before stimulus onset that can be seen in some of the time courses reflect small artifacts originating from the Braille stimulators (see Materials

and Methods).

Consistent with previous human electrophysiological studies using mechanical tactile stimulation, a first brain response (M50) peaked $\sim 50$ ms after stimulus onset in sensors overlying S1 contralateral to the stimulated finger. Source analysis localized this effect into contralateral sensorimotor cortex (supplemental Fig. 1 , available at www.jneurosci.org as supplemental material). It is well established that the M50 response originates from Brodmann area 3b (Elbert et al.,1995; Simões et al., 2001; Braun et al., 2002). Approximately $30 \mathrm{~ms}$ later, an additional more lateralized field pattern became visible. The lateral region corresponds well to the known location of S2. This field topography is in good correspondence with several previous reports (Simões et al., 2003; Palva et al., 2005) that have shown distinct and clearly separable topographies for S1 and S2 on the planar gradient representation.

From $\sim 120$ ms after stimulus onset, neuromagnetic activity becomes increasingly bilateral over somatosensory areas and extends over primarily lateral parietal regions. This topography with two bilateral peaks is sustained over several hundred milliseconds.

Starting from $\sim 300 \mathrm{~ms}$ after stimulus onset, a new cluster of activity in sensors overlying medial occipital cortex emerged. As can be seen in Figure 2, the time courses of the evoked fields measured by these sensors appear to be primarily independent 
from sensors overlying somatosensory and more posterior parietal cortex. This suggests that the signal measured here is not an effect of sensors picking up activity from remote sources (known as common pickup problem) but reflects activity from occipital cortex. Nevertheless, there are other source configurations possible that could produce such a pattern, with the sources not being located in occipital regions. However, in Figure 3, we provide evidence that these signals originate most likely from the medial occipital region. Figure 3, $A$ and $B$, shows the averaged topography in a single subject over the period from 350 to $600 \mathrm{~ms}$ in both planar $(A)$ and axial $(B)$ gradiometer representation, respectively. The local occipital maximum in the planar gradient fields (Fig. $3 A$ ) corresponds to the clear dipolar pattern in the axial gradient fields and thus originates from a medial occipital region (Fig. 3B). Furthermore, in Figure $3 C$, we show the correlation coefficient computed on the time courses in the time interval between 350 and $600 \mathrm{~ms}$ of axial gradiometer signals of the sensor marked in black with those of all other sensors. The strong negative correlation of the positive (blue) side lobe with the marked sensor lying in the center of the negative side lobe, suggesting its antiphase, are strong evidence that this field pattern indeed constitutes a dipolar source located in medial occipital cortex.

Time courses of evoked fields differed between the attended and unattended condition. The earliest significant differences were found $\sim 130$ ms after stimulus onset. However, effects of attention on timelocked activity in the somatosensory system have been described in several previous publications (Hoechstetter et al., 2000; Eimer and Forster, 2003). Because the focus of this study is on oscillatory neuronal synchronization, we will not describe in greater detail attention effects on time-locked activity.

\section{Effects of tactile stimulation in the frequency domain}

In this section, all figures will display $z$ scores that result from the statistical comparison between stimulation and baseline as computed for each time-frequency bin and cumulated across subjects. Because the topographies of frequency-domain effects for left and right finger stimulation were highly symmetrical, the time-frequency representations (TFRs) shown in this section are pooled statistics from left- and right-hand stimulation (against baseline), computed on a selection of sensors (marked in black) overlying the cortex contralateral to the respective side of stimulation (for details, see Fig. 4).

Tactile stimulation resulted in an enhancement of activity in the gamma-frequency range accompanied by a widespread suppression of low-frequency activity, which then rebounded. We will start with the description of the results of the analysis for the high frequencies. The TFRs in Figure 4 show the effect of stimulation from sensors overlying the contralateral somatosensory cortex for each stimulation side. Figure $4 A$ shows the results for the unattended stimulus and Figure $4 B$ for the attended stimulus.

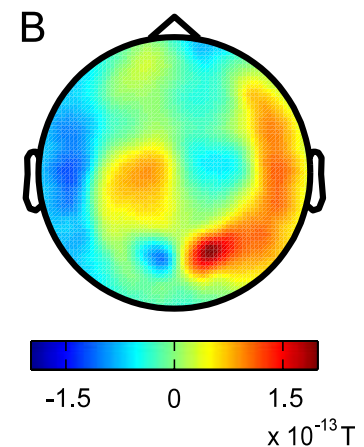

Axial gradient field

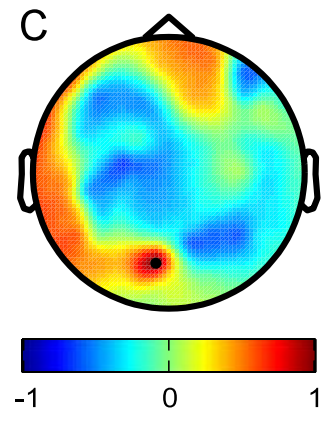

Correlation coefficient
Figure 3. $A$, Topography of the planar gradiometer representation of evoked fields of one example subject in the window
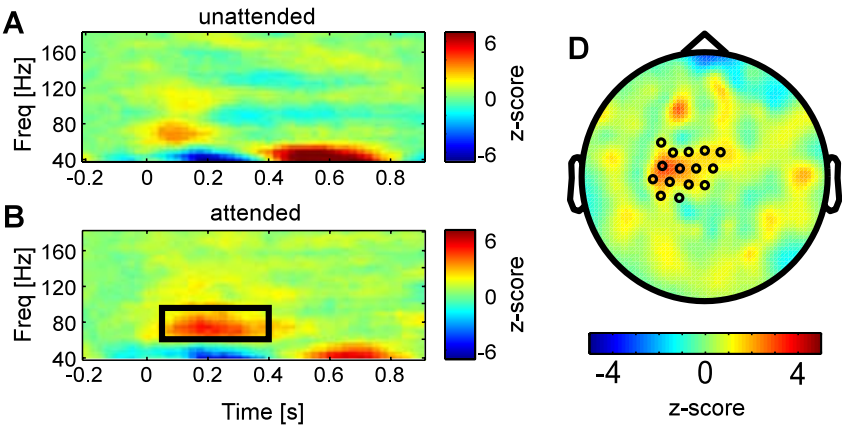

In both conditions, there is an early increase of gamma power lasting until $\sim 200 \mathrm{~ms}$ for unattended stimuli and until $\sim 500 \mathrm{~ms}$ for attended stimuli, respectively. This effect is concentrated in the frequency range between 60 and $95 \mathrm{~Hz}$, a spectral pattern that resembles the pattern known from studies in the visual system. The TFR of evoked fields did not show such a pattern and neither did the phase-locking factor (data not shown). Therefore, this enhancement of gamma power is primarily non-phase locked to the stimulus. Despite the substantially lower signal amplitude for higher frequencies, the effect is very consistent and statistically significant. The strong modulations visible in Figure $4, A$ and $B$, at $\sim 40 \mathrm{~Hz}$ correspond to spectral components that peak at $\sim 20$ $\mathrm{Hz}$ but that become visible at higher frequencies because of their broadband nature and the relatively large spectral concentration $( \pm 10 \mathrm{~Hz})$ used to optimally study the gamma-band activity. The topographies of the stimulus induced gamma-band activity are shown separately for stimulation of the left finger (Fig. 4C) and the right finger (Fig. $4 D$ ). Stimulation of the right finger resulted in an increased gamma-band power in sensors covering early somatosensory cortex over the left hemisphere. When the left finger was stimulated, gamma-band activation peaked over right somatosensory cortex, and there was some additional ipsilateral activation. The maxima of the gamma-band enhancements were localized in the same planar gradiometers as the medial evoked field components between 40 and $100 \mathrm{~ms}$ after stimulus for both stimulation sides. Whether this difference between the effects of right and left finger stimulation is systematic and potentially at- 


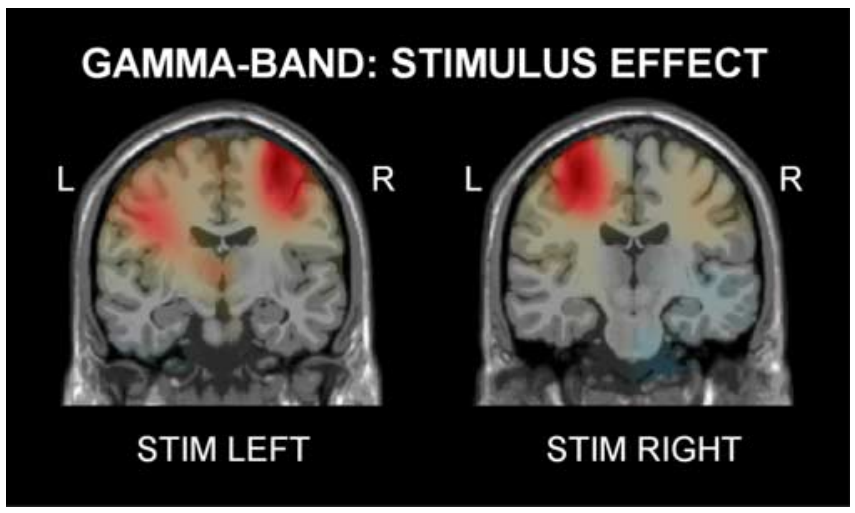

Figure 5. Source analysis of the gamma response as an effect of stimulation versus baseline. Stimulation of the left finger resulted in activation of regions corresponding to right primary somatosensory cortex (right) and vice versa (left). L, Left; R, right.

tributable to the right-handedness of our subjects is unclear. Source analysis ( $z$ scores pooled across eight subjects; for details, see Materials and Methods; one subject was excluded from this analysis because of very low signal-to-noise ratio) revealed one strongly dominant spatial peak of gamma-band activation with a location consistent with primary sensorimotor cortex contralateral to the side of stimulation (Fig. 5). This is symmetrically the case for stimulation of both sides. Similar to the sensor level topography, there was an additional ipsilateral activation for lefthand stimulation.

The resulting source location is highly similar to the one of the M50, which is known to originate from S1. Thus, the source analysis suggests an origin of the gamma-band activity in primary somatosensory cortex. However, the spatial precision of source estimation from MEG data are limited in general and in particular for signals with a moderate to small signal-to-noise ratio as is the case for the gamma-band activity described here. Source analysis alone can in this case not differentiate between different neighboring cortical areas. Beyond spatial location, dipolar sources have another distinguishing feature, namely their orientation. The orientation of a dipole can be directly appreciated from the axial gradiometer topography. We therefore aimed at comparing the axial gradient field topographies of the induced gamma-band activity and of the M50 for an individual subject. Because the signal-to-noise ratio at the single-subject level was not sufficient to do this with the delayed-match-to-sample paradigm, we performed an additional measurement. In two separate sessions with one subject, we applied $>1000$ stimuli each, to either the right or the left index finger. There was no behavioral task or response to minimize the potential for any motor cortical activity. Furthermore, stimulus duration was extended to $300 \mathrm{~ms}$ such that the Braille stimulator operated at its full stimulus amplitude. In Figure $6, A$ and $B$, we show the axial gradiometer topographies of the M50 component of the evoked field. In Figure $6, C$ and $D$, we show the topographies of the mechanical stimulation-related gamma-band activity. The topographies of the M50 and of the gamma-band activities for a given stimulation side are strikingly similar in both position and dipole orientation. This is strong evidence that the gamma-band activity originates from the same source as the M50, i.e., the primary somatosensory cortex.

Also, functional considerations suggest that the observed gamma-band activity originates from somatosensory cortex and in particular is not motor related. (1) We only analyzed trials in which no motor response was given. (2) The observed stimulus-
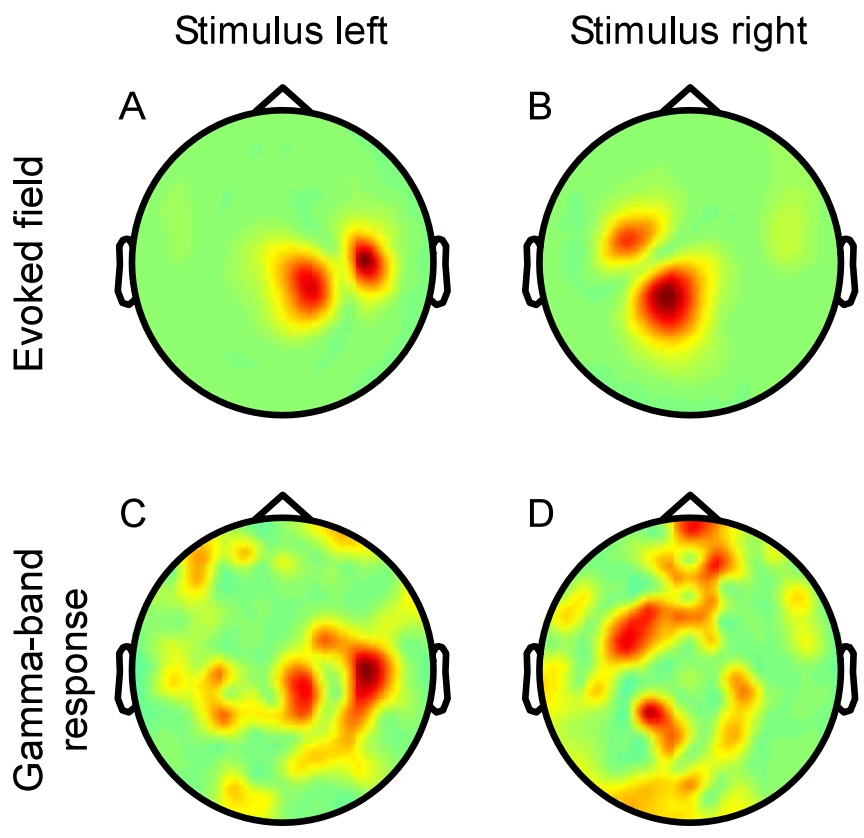

Figure 6. $A, B$, Topography of the squared M50 component, the first peak of the evoked field, $50 \mathrm{~ms}$ after stimulus onset. The evoked field was squared to allow better comparison with the frequency-domain results, because power is also a squared amplitude value. $C, D$, Axial gradiometer topographies of the gamma-band enhancement after left and right finger stimulation. Shown are $z$ values between poststimulus and baseline periods as in Figure 4 .

related gamma-band activity started $\sim 50 \mathrm{~ms}$, and its attentional enhancement (see below) commenced $\sim 100 \mathrm{~ms}$ after stimulus onset. Both effects are therefore substantially earlier than the average behavioral response latencies to target stimuli, which was $\sim 500 \mathrm{~ms}$. Indeed, at the latencies of behavioral responses to target stimuli, the attentional gamma-band enhancement to nontarget stimuli had already ended. (3) The behavioral response to the target was given with both hands, but the sources of gammaband activity showed a strong contralateral dominance.

The most prominent effect of tactile stimulation was a large suppression of low-frequency oscillatory activity (mu and beta band) ranging from $\sim 7 \mathrm{~Hz}$ up to $\sim 40 \mathrm{~Hz}$. This effect was evident in numerous sensors covering a large area of cortex (Fig. 7C,E). The TFRs for the low frequencies are shown in Figure 7 for the sensors overlying contralateral somatosensory cortex and Figure 10 for sensors overlying contralateral occipital cortex (in both cases, again pooled for both stimulation sides). Despite the broadness of the effect, both spatially and spectrally, it had a distinct spatiospectral structure. Low-frequency suppression or desynchronization has a maximum over contralateral somatosensory cortex (Fig. $7 C, E$ ) and a spectral peak at $\sim 20 \mathrm{~Hz}$. The suppression in the beta band has an early onset, which, however, is hard to determine exactly given the low temporal resolution for lower frequencies and the overlapping effects of successive stimuli occurring in close temporal proximity. Suppression reaches its maximum after $250 \mathrm{~ms}$ and lasts until $\sim 400 \mathrm{~ms}$ after stimulus onset (for detailed time courses, see Fig. 12). Note that there is a shift toward lower frequencies over time.

The source reconstruction for the beta suppression (Fig. 8) (comparison: stimulation of unattended finger with baseline; three subjects excluded because of nonstationary baseline) confirms the localization of this effect to primary sensorimotor areas with a strong contralateral bias. This is consistent with reports from previous studies (Crone et al., 1998; Nikouline et al., 2000).

Suppression of mu and beta activity was followed by a re- 
bound in a broad beta band, ranging from $\sim 15$ to $40 \mathrm{~Hz}$ (and thus extending well into the classical gamma range) and lasting until the onset of the next stimulus. The topography shows that the effect is present over bilateral somatosensory/sensorimotor regions (Fig. 7D,F), with a weak emphasis over the hemisphere ipsilateral to the side of stimulation. Source analysis (Fig. 9; comparison: stimulation of unattended finger with baseline; one subject excluded because of nonstationary baseline) revealed a bilateral activation pattern over sensorimotor regions.

Suppression of beta and alpha/mu activity, although being maximal in somatosensory cortex, extends into bilateral occipital regions. The suppression in occipital cortex ranges from $\sim 7.5$ to $30 \mathrm{~Hz}$ and from $\sim 150$ to $400 \mathrm{~ms}$ in the unattended and from 150 to $600 \mathrm{~ms}$ in the attended condition (for details, see Fig. $10 A, B$ ). Note that the topographies in Figure 9 show attention effects, not stimulation effects. Albeit there are similarities of this posterior effect with the suppression over somatosensory areas, the pattern is clearly different with respect to the spectrotemporal dynamics. For the occipital sensors, the spectral distribution has two peaks at 10 and $18 \mathrm{~Hz}$, in contrast to one very broad distribution in the somatosensory system. Also, in occipital sensors, the effect appears to be stronger in the alpha range compared with the beta range. Besides that, in occipital sensors, alpha/beta suppression is particularly strong for attended stimuli and much less prominent for unattended stimuli. Source analysis of the stimulus induced suppression of alphaband activity (Fig. 11) (comparison: stimulation of attended finger with baseline; one subject excluded because of a nonstationary baseline) reveals extended regions of parietal and parietooccipital cortex to be involved. Although the suppression is bilateral, it has a maximum over the hemisphere contralateral to the side of stimulation.

Thus, tactile stimulation results in a combined enhancement of high-frequency oscillations and suppression of low-frequency oscillations in somatosensory cortex. The topographies of highand low-frequency modulations are different in the sense that high-frequency modulations have a more distinct spatial distribution, are evident in sensors overlying slightly more medial cortical areas, and are, at least for the right stimulation side, strictly contralateral. Suppression of lower frequencies, in contrast, show a spatially more widespread and bilateral topography, the maximal effect here being located in slightly more lateral somatosensory/sensorimotor cortex. This is followed by a rebound over bilateral sensorimotor cortex. Furthermore, tactile stimulation resulted in a suppression of low-frequency activity over occipital areas.

Effects of spatial tactile attention in the frequency domain As could already be seen from the figures in the last paragraph, attention modulates oscillations in several frequency bands in different anatomical locations. The most prominent attentional effect in the somatosensory system is the enhancement and prolongation of induced gamma oscillations in contralateral somatosensory cortex. The left column of Figure 12 shows the time courses of the power changes in the frequency bands of interest in more detail, separately for the left and right finger stimulation,
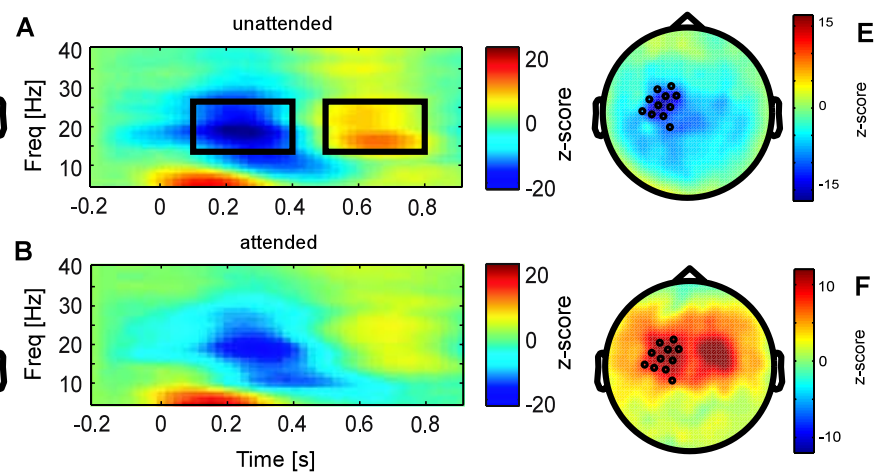

\section{N}

Time $[\mathrm{s}]$

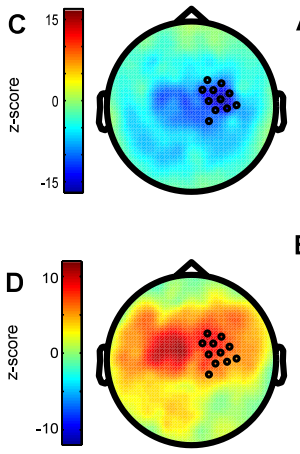

cy resolved power change after stimulation compared with baseline, calculated separately for unattended and attended stimuli over combined somatosensory channels as marked in the topographies to the left and to the window as marked in $\boldsymbol{A}$. $\boldsymbol{D}$, Topography (planar gradients) of beta rebound for left finger stimulation. Averaged over the late $(0.5-0.8 \mathrm{~s})$ time-frequency window as marked in $\boldsymbol{A}$. $\boldsymbol{E}, \boldsymbol{F}$, Topographies of beta suppression and rebound, respectively, for right-hand stimulation. Same time-frequency windows as in $\boldsymbol{C}$ and $\boldsymbol{D}$, respectively. All data are shown in planar gradients.

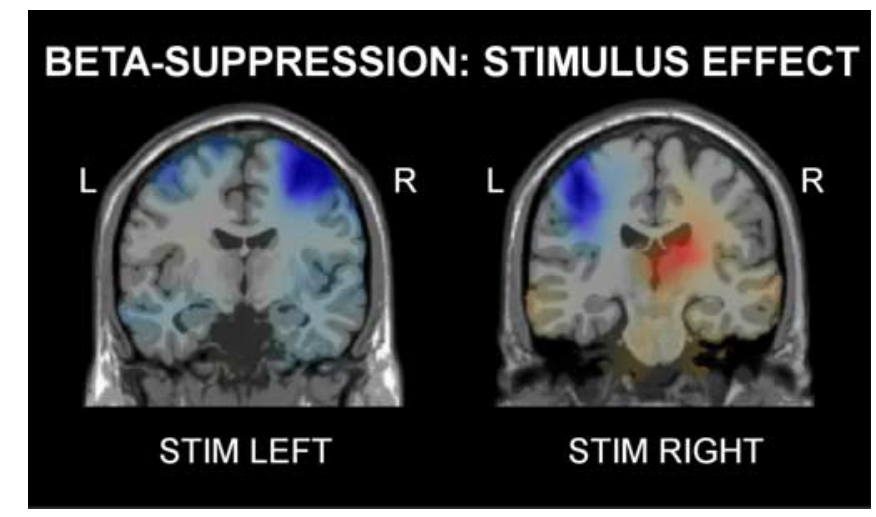

Figure 8. Source analysis of beta suppression as an effect of stimulation versus baseline. Stimulation of the left finger resulted in suppression of beta-band activity in regions corresponding to right primary sensorimotor cortex (left) and vice versa (right). L, Left; $R$, right.

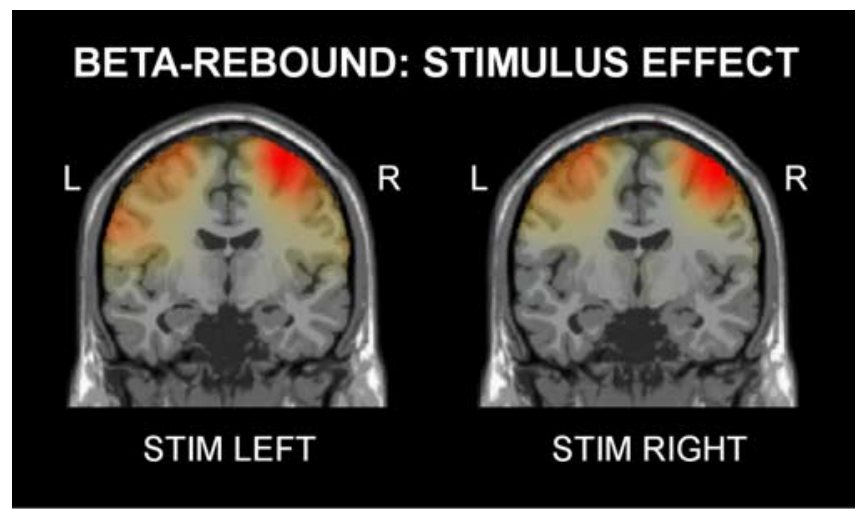

Figure 9. Source analysis of beta-rebound as an effect of stimulation versus baseline. Stimulation of either finger resulted in bilateral rebound in sensorimotor areas with a slight dominance over the right hemisphere. L, Left; $R$, right.

averaged over those sensors marked in the respective topographies in Figures 4, 7, and 10. To show the consistency of the effects, the right column of Figure 12 shows scatter plots of power changes after stimulation in individual subjects in the time-frequency windows and sensors as marked in the respective figures (Figs. 4, 7, 10). Power changes for attended stimuli are plotted on the $y$-axis, power changes for unattended stimuli are plotted on 

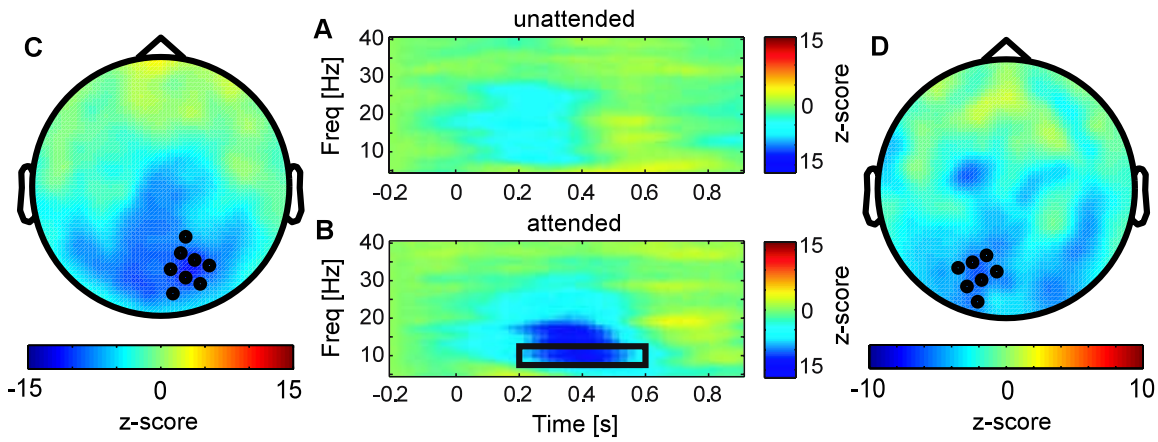

Figure 10. $\quad \boldsymbol{A}, \boldsymbol{B}$, Time-frequency resolved power change after stimulation compared with baseline calculated separately for unattended and attended stimuli over combined occipital channels as marked in the topographies to the left and to the right ( $\boldsymbol{C}$ D). C, Topography of the attentional effect on alpha suppression in the marked time-frequency window. Note that this is different from the previous figures in which stimulation effects were shown. $D$, Topography of the attentional effect on alpha suppression in the marked time-frequency window. All data are shown in planar gradients.

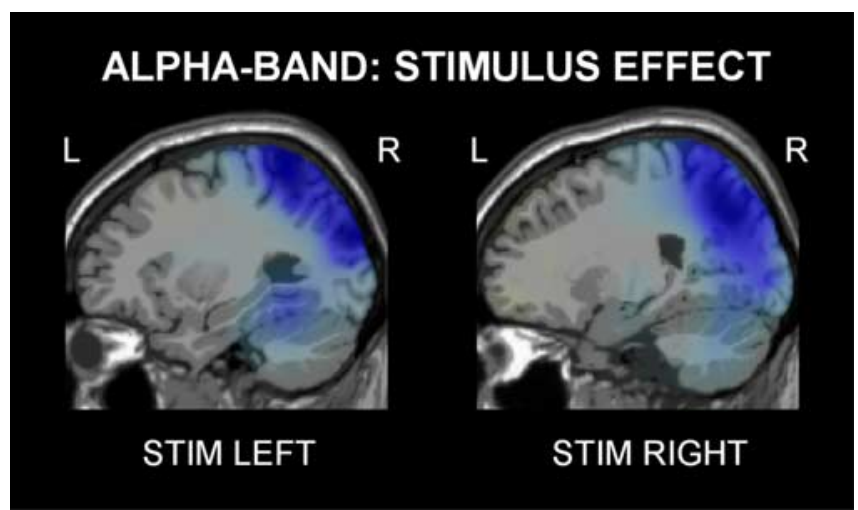

Figure 11. Source analysis of alpha suppression as an effect of stimulation versus baseline. Stimulation of either finger resulted in bilateral suppression of alpha-band activity in parietooccipital cortex. L, Left; $R$, right.

the $x$-axis, and, therefore, values lying left above the diagonal indicate an increase of power for the attended compared with the unattended stimuli, whereas values in the field right below the diagonal mean a decrease. Please note that the same time-frequency window and group of sensors was used for all subjects, and, because both the spectrotemporal and spatial pattern of these effects vary across subjects, this will underestimate the true strength of the effect of tactile stimulation.

Gamma-frequency oscillations $(60-95 \mathrm{~Hz})$ to attended stimuli, although having the same onset as for the unattended stimuli, continued to rise further when, $\sim 100 \mathrm{~ms}$ after stimulus onset, power started to decrease again after presentation of an unattended stimulus. This was consistently the case for left and right finger stimulation, and gamma power was then substantially enhanced in a window ranging from $\sim 100$ to $500 \mathrm{~ms}$ after stimulus onset (Fig. 12). The statistical comparison (paired $t$ test) over the time-frequency window from 50 to $400 \mathrm{~ms}$ and 60 to $95 \mathrm{~Hz}$ revealed the significance of this effect $(t=1.86$ and $t=2.75, p<$ 0.05 , for right and left-hand stimulation, respectively), and this is also evident from the two scatter plots in the top right panel: all but one of the subjects show the effect consistently for left and right finger stimulation. The source analysis for the effect of attention on gamma-band activity (Fig. 13) revealed that the location of this attention effect colocalizes with the respective stimulation effect. This held for both stimulation sides, representing independent datasets. Thus, we conclude that attention consis- tently enhances gamma oscillations over contralateral primary somatosensory cortex.

Equivalent tests were performed for all frequency bands and time windows described in the last section. The attention contrast for the suppression of the $\mathrm{mu}$ rhythm, which was performed over a window from 0.2 to $0.6 \mathrm{~s}$ and from 7.5 to 12.5 $\mathrm{Hz}$ did not reveal any significant difference $(t=-0.58$ and $-1.46, p>0.05$ for right and left hand, respectively). The results of the statistical comparison for the beta suppression were more complicated. At first glance, the beta suppression appeared to be significantly reduced for attended stimuli (time- and frequency-range tested: 0.1$0.4 \mathrm{~s}$ and $13.5-26.5 \mathrm{~Hz} ; t=1.98$ and $t=$ 3.94; $p<0.05$ and $p<0.01$ for right and left hand, respectively). However, this difference is mainly attributable to the interaction of two effects: (1) the beta rebound lasted longer than the interstimulus interval, and (2) the beta rebound was reduced contralateral to the attended side (see below). As a result of those two effects, the prestimulus baseline in the beta band was reduced contralateral to the attended side $(t=-2.58$ and $t=-2.34, p<$ 0.05 for right and left hand). This reduced baseline explained the described apparent reduction in the beta decrease after stimulation. Therefore, we did a separate analysis in which only the data from the first test stimulus presentation (which was not immediately preceded by a tactile stimulus and thus had a stationary baseline) was included. In this analysis, there was no significant attention effect for the beta and mu suppression $(t=-1.01$ and $t=-0.91, p>0.1$ for beta band; and $t=-0.53$ and $t=-0.28$, $p>0.1$ for mu band, values for right and left hand, respectively). In Figure 12, we show the time courses for the somatosensory beta band from the analysis that was restricted to the data from the first stimulus presentation and provide the time courses of beta power for all test stimuli in supplemental Figure 2 (available at www.jneurosci.org as supplemental material). Although attention did not affect the beta decrease, it resulted in a robust delay and suppression of the beta rebound selectively over contralateral somatosensory cortex. This suppression of the beta rebound was found in both analyses, the one calculated over the data only involving the first test stimulus as well as the one that was done across all. Statistics were computed for the window from 13.5 to $26.5 \mathrm{~Hz}$ and from 0.5 to $0.8 \mathrm{~s}$ after stimulus onset. The effect was significant for both the left and the right stimulation side $(t=$ -1.88 and $t=-1.88 ; p<0.05)$. The scatter plot in the second row of Figure 12 shows that this effect is also quite consistent over individual subjects. Source analysis of the attentional effect (Fig. 14) shows that, whereas the beta rebound itself had a bilateral topography, the attentional suppression of the beta rebound is primarily restricted to the hemisphere contralateral to the side of stimulation and covers a large portion of the sensorimotor strip.

Finally, attention deepened the suppression of alpha and beta power over occipital sensors. The effect was maximal over occipital cortex as displayed in the topographies of Figure 10, $C$ and $D$. Note that these topographies, unlike those in Figures 4 and 7, do not show the stimulation effect but show the statistical difference ( $z$ value) between attended and unattended conditions in the time-frequency window, as indicated in Figure 10B. Although the stimulus-induced suppression was maximal over somatosensory cortex (similar topographies as in Fig. 7C,E), the effect of 
attention was maximal over contralateral occipital areas. The time courses and scatter plots for the occipital channels can be seen in the bottom two rows of Figure 12. Attention distinctly prolonged and enhanced alpha and beta suppression over bilateral occipital cortex, with an emphasis over the hemisphere contralateral to the stimulation side. Statistical comparisons for the effect of attention were computed for the following time-frequency-windows: (1) beta band: $13.5-26.5 \mathrm{~Hz}, 0.1-0.4$ $\mathrm{s}$ (same as for somatosensory system), resulting in a statistically significant reduction of beta power in the attended compared with the nonattended condition for both hands $(t=-2.16, p<0.05$; $t=$ -3.33, $p<0.01$, for right and left hand, respectively); (2) alpha band: $7.5-12.5 \mathrm{~Hz}$, $0.2-0.6 \mathrm{~s}$, resulting in a statistically significant reduction of alpha power in the attended compared with the nonattended condition for both hands $(t=-2.96$ and $t=-3.23, p<0.01$, for right and left hand, respectively).

Source analysis (Fig. 15) shows that the attentional suppression includes occipital areas more inferior than those involved in the stimulus-induced suppression. The source reconstruction did not reveal a clear lateralization of the effect, although that was statistically significant in the sensor data. Rather, it showed a bias toward the left hemisphere, independent of the side of stimulation.

To summarize, using mechanical stimulation and MEG recordings, we obtained physiologically meaningful brain responses that are consistent with the known anatomical locations for both evoked fields and the mu and beta suppression (or event-related desynchronization). Attending to tactile stimuli strongly and consistently enhances gamma oscillations in the high-frequency range while not modulating (or modulating only slightly) the suppressive effects of tactile stimulation on low-frequency activity in somatosensory areas. In contrast, there is a strong and consistent attentional deepening of alpha and beta suppression over occipital cortex. Furthermore, attending to a finger also has a late effect and suppresses the bilateral beta rebound particularly over contralateral somatosensory cortex.

\section{Discussion}

We investigated the effects of tactile stimulation and spatialselective tactile attention on oscillatory neuronal activity in humans. We found an early stimulus-induced, but not stimuluslocked, gamma-band response $(60-95 \mathrm{~Hz})$. The onset latency, the strong contralateral dominance, and the source reconstruction strongly suggest an origin in primary somatosensory cortex. This is consistent with other recent studies that have reported
Gamma-band (60 - $95 \mathrm{~Hz}$ ) power change - somatosensory channels

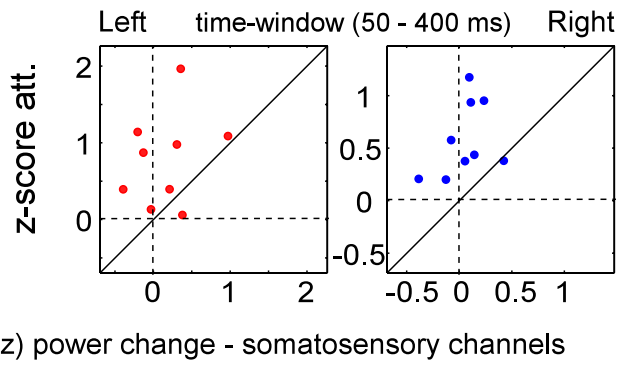

Beta-band $(14-26 \mathrm{~Hz})$ power change - somatosensory channels
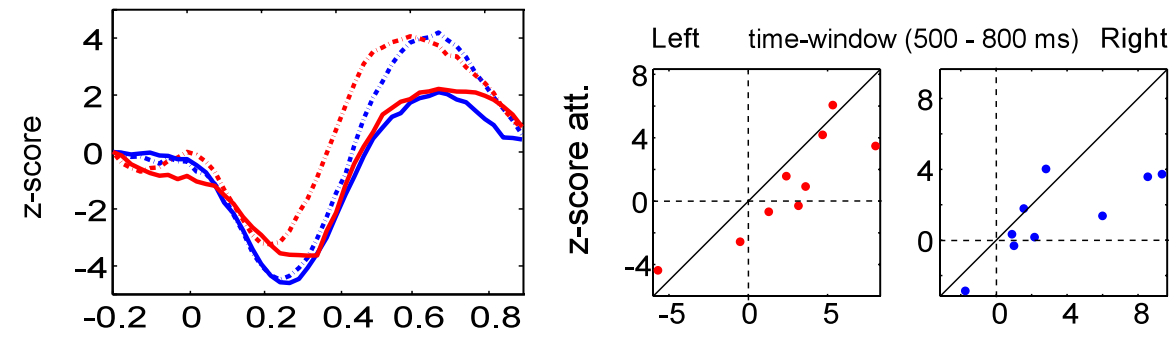

Beta-band (14 - $26 \mathrm{~Hz}$ ) power change - occipital channels
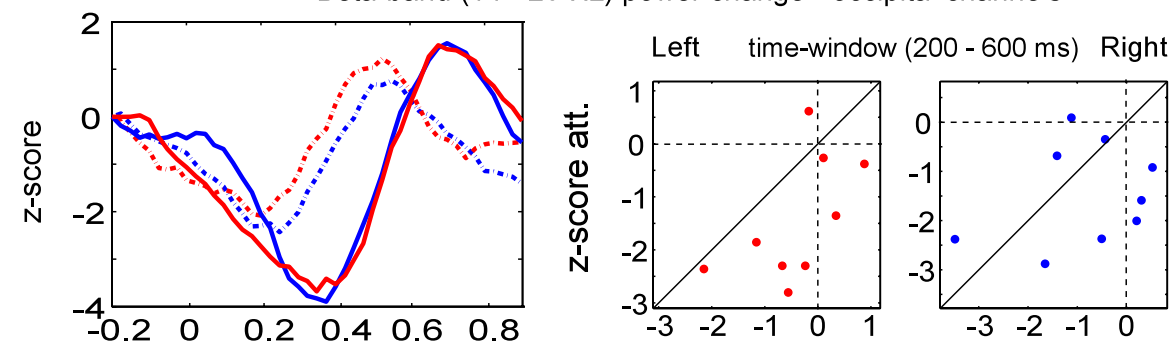

Alpha-band $(7.5-12.5 \mathrm{~Hz})$ power change - occipital channels
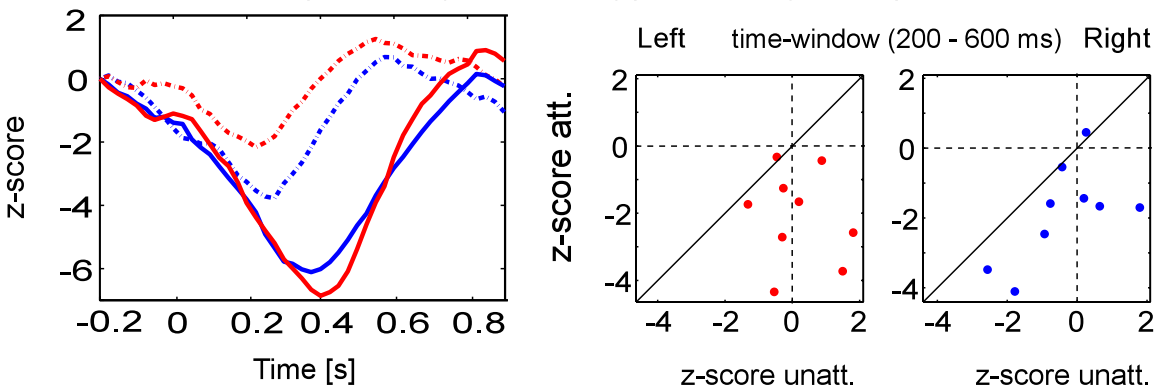

Figure 12. Left column, Time courses of the stimulation-induced power changes for different frequency bands in the group of channels (contralateral to stimulation) as indicated in Figures 4, 6, and 9 separately for right- and left-hand stimulation. Right column, Scatter plots showing individual subjects' data of stimulation-induced power changes in the respective frequency bands for unattended and attended stimuli as measured over the depicted channels and time-frequency windows (see Figs. 4, 6, 9). gamma-band activity in the human primary somatosensory cortex recorded with MEG (Hirata et al., 2002; Gaetz and Cheyne, 2003; Ihara et al., 2003). Those previous studies, however, used electrical median nerve stimulation in contrast to mechanical stimulation as used here. MEG and EEG activity to electrical stimuli have different characteristics, such as the presence of very short latency responses in early somatosensory areas (e.g., N20m-P35) (Simões et al., 2003) that are absent in our data and those of others using mechanical stimulation of the skin (Simões et al., 2001; Braun et al., 2002).

We found that stimulus-induced gamma-band activity in somatosensory cortex is strongly enhanced and prolonged by spatial tactile attention. Source reconstruction revealed colocaliza- 


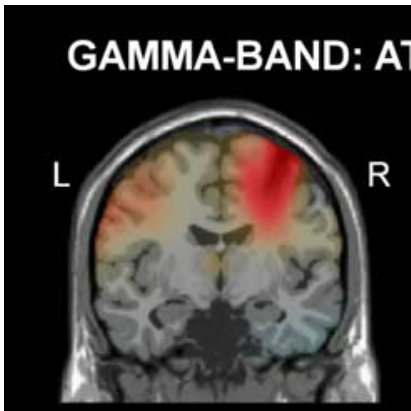

STIM LEFT

\section{TENTION EFFECT}

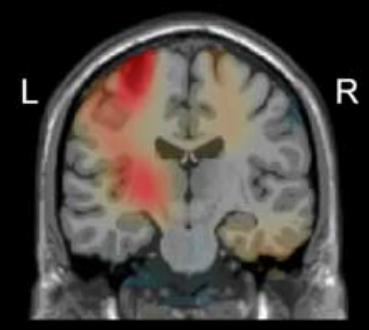

STIM RIGHT
Figure 13. Source analysis of the effect of attention on gamma-band activity. Attention to the left finger resulted in enhanced gamma-band activity in right somatosensory cortex (left) and vice versa (right). L, Left; $R$, right.

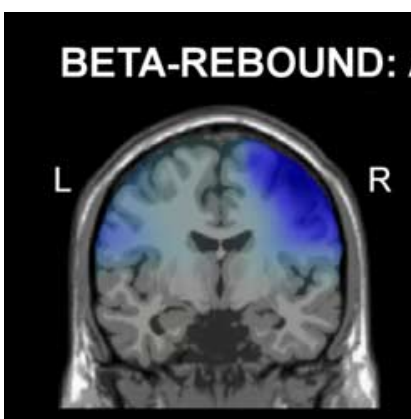

STIM LEFT

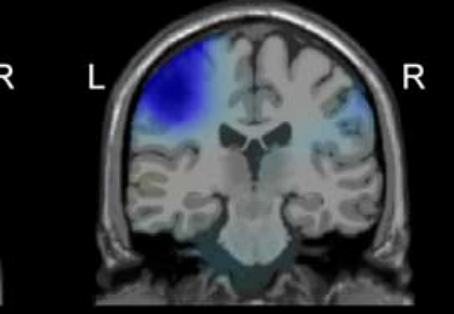

STIM RIGHT
Figure 14. Source analysis of the effect of attention on the beta rebound. Attention to the left finger resulted in a reduced beta rebound in right sensorimotor cortex (left) and vice versa (right). L, Left; R, right.

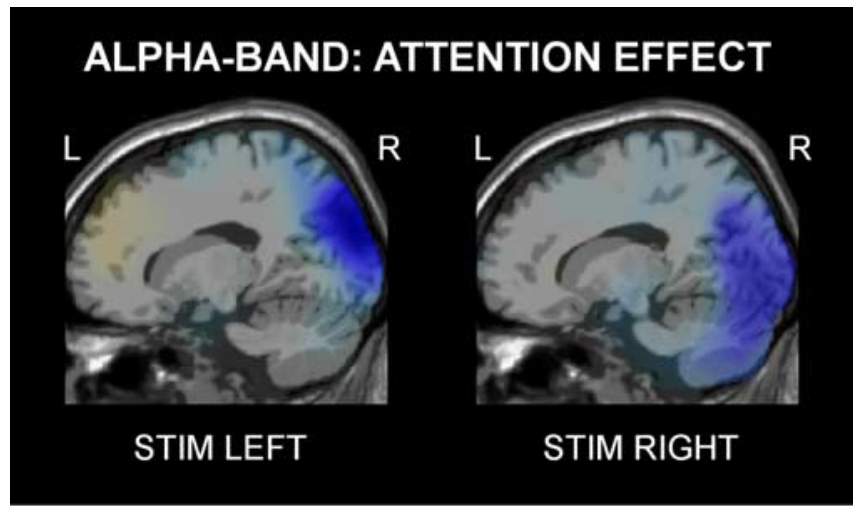

Figure 15. Source analysis of the effect of attention on alpha suppression. Stimulation of either finger resulted in bilateral suppression of alpha oscillations in parieto-occipital and occipital cortex.

tion of this attentional enhancement with the effect of stimulation in contralateral S1. This result is in contrast to findings from other electrophysiological studies assessing spike rates or event-related potentials/fields that have generally found attentional effects to be stronger in S2 and rather weak or absent in S1 (Burton and Sinclair, 2000a; Hoechstetter et al., 2000) (but see Braun et al., 2002). However, functional imaging studies, relying on hemodynamic signals, did reveal attention effects in S1 (for review, see Burton and Sinclair, 2000b). It is by now well estab- lished that the hemodynamic response is more tightly correlated with synchronized gamma-band activity than with neuronal firing rates (Logothetis et al., 2001; Niessing et al., 2005). Therefore, one possible explanation for the apparently conflicting results from the different approaches is that attention synchronizes gamma-band oscillations in primary somatosensory cortex, which translates into enhanced spiking activity at later processing stages. We will further discuss this point at the end of Discussion.

The attentional enhancement in this study commenced $\sim 100$ $\mathrm{ms}$ and lasted until $\sim 500 \mathrm{~ms}$ after stimulus onset. The effect was found in the data of eight of nine subjects, and the time courses of gamma-band activity were highly overlapping for stimulation of either hand. Among the early responses in the somatosensory system, the attentional effect on the induced gamma-band activity was stronger and more consistent than attentional effects on oscillatory activity in other frequency bands or on stimuluslocked components. This suggests that gamma-band oscillations play an important functional role in the early stages of the somatosensory system for processing behaviorally relevant stimuli. We hypothesize that gamma-band synchronization in primary somatosensory cortex is instrumental in communicating with higher somatosensory areas.

Although tactile stimulation induced gamma-band activity, it also led to the well known suppression of the somatosensory mu $(\approx 8-15 \mathrm{~Hz})$ and beta $(\approx 15-25 \mathrm{~Hz})$ activity (Crone et al., 1998; Pfurtscheller et al., 2002) and the subsequent beta rebound ( $\approx 15-25 \mathrm{~Hz}$ ) (van Burik and Pfurtscheller, 1999). Consistent with some of the previous studies, mu and beta suppression are widespread in both their spectral distribution (ranging from 7 to $40 \mathrm{~Hz}$ ) and spatial distribution, peaking over somatosensory/ sensorimotor cortex contralateral to the stimulated hand but also reaching to ipsilateral sensorimotor cortex and bilateral parietal and occipital regions. Stimulus-induced modulations of mu- and beta-band activity were of very high signal-to-noise ratio. Nevertheless, there was no significant modulation of the somatosensory mu and beta suppression with spatial-selective attention. This suggests that the respective desynchronization phenomena are not directly involved in the mechanisms underlying the attentional selection of behaviorally relevant stimulus information. Rather, they appear to reflect an automatic response of the sensorimotor circuit to afferent stimulation that occurs independently of processing demands. In contrast, there was a significant reduction of the beta rebound with spatial-selective attention. This attentional effect started $\sim 500 \mathrm{~ms}$ after onset of the nontarget stimuli, whereas the average reaction time to the target stimuli was in the order of $510 \mathrm{~ms}$. Therefore, a direct functional role of the attentional modulation of the beta rebound for sensory processing or decision making in the somatosensory system has to be considered unlikely.

An interesting attentional effect was found for the occipital alpha-band (7.5-12.5 Hz) and beta-band (13.5-26.5 Hz) activity. Attended tactile stimuli resulted in a deeper and longer-lasting suppression of occipital alpha and beta-band activity than unattended tactile stimuli (Eimer, 2000; Worden et al., 2001; Kennett et al., 2003). Furthermore, tactile stimulation did not only alter ongoing oscillatory activity in occipital cortex but also resulted in stimulus-locked activation over occipital cortex. Evoked fields showed a clear spatial peak over medial occipital cortex, with an onset latency of $\sim 350 \mathrm{~ms}$. There was no consistent attentional modulation of this component, in contrast to the strong attentional modulation of the stimulus-induced occipital alpha and beta suppression. This suggests different functional mechanisms behind those two processes. 
Previous hemodynamic studies have shown that tactile stimulation can activate various parts of visual cortex in blind (Sadato et al., 1996) and normally sighted (Zangaladze et al., 1999; Amedi et al., 2002) human subjects. In principle, this might be related to direct input from heteromodal areas (Falchier et al., 2002; Schroeder and Foxe, 2002) or to mechanisms underlying visual imagery that are not yet fully understood (Zhang et al., 2004). The tactile evoked fields that we found over occipital areas showed a late onset. We therefore consider it unlikely that they were attributable to direct input from early somatosensory areas.

One potential concern is that physical stimulation might not have been identical for the two attention conditions because attention might have invoked motor programs to optimize haptic sensing. For example, subjects might have made exploratory movements or pushed the attended finger slightly stronger onto the Braille cells than the unattended finger. For several reasons, we are convinced that this is not the case. First, stimulation was very short, lasting only $35 \mathrm{~ms}$ and thereby excluded the possibility that subjects engaged in active exploration of the stimuli. Second, the time courses of gamma-band activity and evoked fields are identical in the two attention conditions until $\sim 100 \mathrm{~ms}$ after stimulus. Third, pushing stronger on the attended side does not seem to be a good strategy because the Braille stimulators use only weak forces to drive the pins and are designed to give optimal sensation when the finger is resting relaxed on them. The pins give a diminished tactile sensation when pushed on or when the finger is lifted off from them. Thus, we can almost certainly rule out that differences in physical stimulation account for the attentional effects reported here.

The mechanisms underlying the observed attention effects are still unknown, and we can only speculate at this point. Neuronal networks have typically been found to show enhanced gammaband activity during activation (Tiesinga et al., 2001; Cunningham et al., 2003). Thus, the attentional enhancement of gammaband activity observed here might be attributable to excitatory input from, for example, a frontoparietal network responsible for the allocation of spatial attention (Corbetta and Shulman, 1998). Alternatively or in addition, top-down input might act through neuromodulators. Acetylcholine seems to support the generation of local gamma-frequency synchronization (Munk et al., 1996; Fellous et al., 2001; Rodriguez et al., 2004) and also the propensity of neurons to be entrained by rhythmic input (Schreiber et al., 2004). Such modulatory input might be of corticocortical or subcortico-cortical nature, but it would have to possess at least a crude spatial selectivity to explain the present results. There might also be top-down input to the neurons processing the unattended stimulus. This input might activate local inhibitory networks to decrease the activation of those neurons. Increased activation of inhibitory networks leads to enhanced beta-band synchronization (Jensen et al., 2005).

The effect of selective attention on stimulus-related gammaband activity was mainly restricted to somatosensory cortex. Many previous studies have reported the activation of other areas during the performance of attention tasks, in particular areas of the frontoparietal attention network (Corbetta and Shulman, 1998). Those different findings are fully compatible with each other. The attention network is typically activated during cue processing and during the allocation of attention (Giesbrecht et al., 2003). In our paradigm, the investigation of this network would have required the comparison between task-related activity and a precue baseline. Rather, we focused here on the effects of selective attention on stimulus-related activity. To this end, we performed direct comparisons between the two attention condi- tions for time-frequency windows with the strongest stimulusrelated activations. The analysis of oscillatory synchronization in the attention network and its interaction with oscillatory synchronization in sensory areas is of great interest but goes beyond the scope of this study.

To summarize, spatial-selective tactile attention enhances and prolongs gamma-band activity in primary somatosensory cortex. This will likely enhance the impact of the activated neurons on their postsynaptic targets, e.g., in secondary somatosensory cortex (Salinas and Sejnowski, 2001; Fries, 2005). Intracellular recordings in visual cortex in vivo have revealed recently that postsynaptic target neurons primarily respond to rapid fluctuations in their synaptic input (Azouz and Gray, 2003). Thus, a neuronal group providing input may enhance its impact through either synchronizing spikes in the gamma band or producing more spikes in the gamma band. In both cases, the MEG power in the gamma band would be enhanced. Investigations using microelectrode recordings of spikes and local field potentials showed that local field potential power can be enhanced in the absence of changes in firing rates but the presence of changes in local synchronization (Fries et al., 2001b). Increases in gamma-band activity in S1 will likely lead to generally increased activation of S2 and thereby might explain some of the attentional effects found in S2. Furthermore, it is conceivable that attention generally renders the reciprocal information exchange between different parts of the neural network, such as S1 and S2, more effective through a stronger oscillatory coupling (Fries, 2005; Schoffelen et al., 2005). These questions will be explicitly addressed in future experiments.

\section{References}

Amedi A, Jacobson G, Hendler T, Malach R, Zohary E (2002) Convergence of visual and tactile shape processing in the human lateral occipital complex. Cereb Cortex 12:1202-1212.

Azouz R, Gray CM (2003) Adaptive coincidence detection and dynamic gain control in visual cortical neurons in vivo. Neuron 37:513-523.

Bastiaansen MC, Knösche TR (2000) Tangential derivative mapping of axial MEG applied to event-related desynchronization research. Clin Neurophysiol 111:1300-1305.

Braun C, Haug M, Wiech K, Birbaumer N, Elbert T, Roberts LE (2002) Functional organization of primary somatosensory cortex depends on the focus of attention. NeuroImage 17:1451-1458.

Brefczynski JA, DeYoe EA (1999) A physiological correlate of the "spotlight” of visual attention. Nat Neurosci 2:370-374.

Burton H, Sinclair RJ (2000a) Tactile-spatial and cross-modal attention effects in the primary somatosensory cortical areas $3 b$ and 1-2 of rhesus monkeys. Somatosens Motor Res 17:213-228.

Burton H, Sinclair RJ (2000b) Attending to and remembering tactile stimuli: a review of brain imaging data and single-neuron responses. J Clin Neurophysiol 17:575-591.

Burton H, Sinclair RJ, Hong SY, Pruett JR, Whang KC (1997) Tactile-spatial and cross-modal attention effects in the second somatosensory and $7 \mathrm{~b}$ cortical areas of rhesus monkeys. Somatosens Motor Res 14:237-267.

Corbetta M, Shulman GL (1998) Human cortical mechanisms of visual attention during orienting and search. Philos Trans R Soc Lond B Biol Sci 353:1353-1362.

Crone NE, Miglioretti DL, Gordon B, Sieracki JM, Wilson MT, Uematsu S, Lesser RP (1998) Functional mapping of human sensorimotor cortex with electrocorticographic spectral analysis. I. Alpha and beta eventrelated desynchronization. Brain 121:2271-2299.

Cunningham MO, Davies CH, Buhl EH, Kopell N, Whittington MA (2003) Gamma oscillations induced by kainate receptor activation in the entorhinal cortex in vitro. J Neurosci 23:9761-9769.

Efron B, Tibshirani RJ (1993) An introduction to the bootstrap. Boca Raton, FL: Chapman and Hall/CRC.

Eimer M (2001) Crossmodal links in spatial attention between vision, audition, and touch: evidence from event-related brain potentials. Neuropsychologia 39:1292-1303. 
Eimer M, Forster B (2003) Modulations of early somatosensory ERP components by transient and sustained spatial attention. Exp Brain Res 151:24-31.

Elbert T, Junghofer M, Scholz B, Schneider S (1995) The separation of overlapping neuromagnetic sources in first and second somatosensory cortices. Brain Topogr 7:275-282.

Engel AK, Fries P, Singer W (2001) Dynamic predictions: oscillations and synchrony in top-down processing. Nat Rev Neurosci 2:704-716.

Falchier A, Clavagnier S, Barone P, Kennedy H (2002) Anatomical evidence of multimodal integration in primate striate cortex. J Neurosci 22:5749-5759.

Fellous JM, Houweling AR, Modi RH, Rao RP, Tiesinga PH, Sejnowski TJ (2001) Frequency dependence of spike timing reliability in cortical pyramidal cells and interneurons. J Neurophysiol 85:1782-1787.

Fries P (2005) A mechanism for cognitive dynamics: neuronal communication through neuronal coherence. Trends Cogn Sci 9:474-480.

Fries P, Roelfsema PR, Engel AK, König P, Singer W (1997) Synchronization of oscillatory responses in visual cortex correlates with perception in interocular rivalry. Proc Natl Acad Sci USA 94:12699-12704.

Fries P, Neuenschwander S, Engel AK, Goebel R, Singer W (2001a) Rapid feature selective neuronal synchronization through correlated latency shifting. Nat Neurosci 4:194-200.

Fries P, Reynolds JH, Rorie AE, Desimone R (2001b) Modulation of oscillatory neuronal synchronization by selective visual attention. Science 291:1560-1563.

Fries P, Schröder JH, Roelfsema PR, Singer W, Engel AK (2002) Oscillatory neuronal synchronization in primary visual cortex as a correlate of stimulus selection. J Neurosci 22:3739-3754.

Gaetz WC, Cheyne DO (2003) Localization of human somatosensory cortex using spatially filtered magnetoencephalography. Neurosci Lett 340:161-164.

Giesbrecht B, Woldorff MG, Song AW, Mangun GR (2003) Neural mechanisms of top-down control during spatial and feature attention. NeuroImage 19:496-512.

Gross J, Kujala J, Hämäläinen M, Timmermann L, Schnitzler A, Salmelin R (2001) Dynamic imaging of coherent sources: Studying neural interactions in the human brain. Proc Natl Acad Sci USA 98:694-699.

Groves PM, Eason RG (1969) Effects of attention and activation on the visual evoked cortical potential and reaction time. Psychophysiology 5:394-398.

Gruber T, Müller MM, Keil A, Elbert T (1999) Selective visual-spatial attention alters induced gamma band responses in the human EEG. Clin Neurophysiol 110:2074-2085.

Hillyard SA, Hink RF, Schwent VL, Picton TW (1973) Electrical signs of selective attention in the human brain. Science 182:177-180.

Hirata M, Kato A, Taniguchi M, Ninomiya H, Cheyne D, Robinson SE, Maruno M, Kumura E, Ishii R, Hirabuki N, Nakamura H, Yoshimine T (2002) Frequency-dependent spatial distribution of human somatosensory evoked neuromagnetic fields. Neurosci Lett 318:73-76.

Hoechstetter K, Rupp A, Meinck HM, Weckesser D, Bornfleth H, Stippich C, Berg P, Scherg M (2000) Magnetic source imaging of tactile input shows task-independent attention effects in SII. NeuroReport 11:2461-2465.

Hsiao SS, O'Shaughnessy DM, Johnson KO (1993) Effects of selective attention on spatial form processing in monkey primary and secondary somatosensory cortex. J Neurophysiol 70:444-447.

Ihara A, Hirata M, Yanagihara K, Ninomiya H, Imai K, Ishii R, Osaki Y, Sakihara K, Izumi H, Imaoka H, Kato A, Yoshimine T, Yorifuji S (2003) Neuromagnetic gamma-band activity in the primary and secondary somatosensory areas. NeuroReport 14:273-277.

Jensen O, Goel P, Kopell N, Pohja M, Hari R, Ermentrout B (2005) On the human sensorimotor-cortex beta rhythm: sources and modeling. NeuroImage 26:347-355.

Kennett S, Eimer M, Spence C, Driver J (2001) Tactile-visual links in exogenous spatial attention under different postures: convergent evidence from psychophysics and ERPs. J Cogn Neurosci 13:462-478.

Knösche TR (2002) Transformation of whole-head MEG recordings between different sensor positions. Biomed Tech (Berl) 47:59-62.

Lachaux JP, Rodriguez E, Martinerie J, Varela FJ (1999) Measuring phase synchrony in brain signals. Hum Brain Mapp 8:194-208.

Logothetis NK, Pauls J, Augath M, Trinath T, Oeltermann A (2001) Neurophysiological investigation of the basis of the fMRI signal. Nature 412:150-157.
Mangun GR, Hinrichs H, Scholz M, Mueller-Gaertner HW, Herzog H Krause BJ, Tellman L, Kemna L, Heinze HJ (2001) Integrating electrophysiology and neuroimaging of spatial selective attention to simple isolated visual stimuli. Vision Res 41:1423-1435.

Meftah el-M, Shenasa J, Chapman CE (2002) Effects of a cross-modal manipulation of attention on somatosensory cortical neuronal responses to tactile stimuli in the monkey. J Neurophysiol 88:3133-3149.

Mitra PP, Pesaran B (1999) Analysis of dynamic brain imaging data. Biophys J 76:691-708.

Munk MH, Roelfsema PR, König P, Engel AK, Singer W (1996) Role of reticular activation in the modulation of intracortical synchronization. Science 272:271-274.

Niessing J, Ebisch B, Schmidt KE, Niessing M, Singer W, Galuske RA (2005) Hemodynamic signals correlate tightly with synchronized gamma oscillations. Science 309:948-951.

Nikouline VV, Linkenkaer-Hansen K, Wikström H, Kesäniemi M, Antonova EV, Ilmoniemi RJ, Huttunen J (2000) Dynamics of mu-rhythm suppression caused by median nerve stimulation: a magnetoencephalographic study in human subjects. Neurosci Lett 294:163-166.

Oldfield RC (1971) The assessment and analysis of handedness: the Edinburgh inventory. Neuropsychologia 9:97-113.

Palva S, Linkenkaer-Hansen K, Naatanen R, Palva JM (2005) Early neural correlates of conscious somatosensory perception. J Neurosci 25:5248-5258.

Pfurtscheller G, Woertz M, Müller G, Wriessnegger S, Pfurtscheller K (2002) Contrasting behavior of beta event-related synchronization and somatosensory evoked potential after median nerve stimulation during finger manipulation in man. Neurosci Lett 323:113-116.

Reynolds JH, Chelazzi L, Desimone R (1999) Competitive mechanisms subserve attention in macaque areas V2 and V4. J Neurosci 19:1736-1753.

Rodriguez R, Kallenbach U, Singer W, Munk MH (2004) Short- and longterm effects of cholinergic modulation on gamma oscillations and response synchronization in the visual cortex. J Neurosci 24:10369-10378.

Sadato N, Pascual-Leone A, Grafman J, Ibanez V, Deiber MP, Dold G, Hallett M (1996) Activation of the primary visual cortex by Braille reading in blind subjects. Nature 380:526-528.

Salinas E, Sejnowski TJ (2001) Correlated neuronal activity and the flow of neural information. Nat Rev Neurosci 2:539-550.

Schoffelen JM, Oostenveld R, Fries P (2005) Neuronal coherence as a mechanism of effective corticospinal interaction. Science 308:111-113.

Schreiber S, Fellous JM, Tiesinga P, Sejnowski TJ (2004) Influence of ionic conductances on spike timing reliability of cortical neurons for suprathreshold rhythmic inputs. J Neurophysiol 91:194-205.

Schroeder CE, Foxe JJ (2002) The timing and laminar profile of converging inputs to multisensory areas of the macaque neocortex. Brain Res Cogn Brain Res 14:187-198.

Simões C, Mertens M, Forss N, Jousmäki V, Lütkenhöner B, Hari R (2001) Functional overlap of finger representations in human SI and SII cortices. J Neurophysiol 86:1661-1665.

Simões C, Jensen O, Parkkonen L, Hari R (2003) Phase locking between human primary and secondary somatosensory cortices. Proc Natl Acad Sci USA 100:2691-2694.

Steinmetz PN, Roy A, Fitzgerald PJ, Hsiao SS, Johnson KO, Niebur E (2000) Attention modulates synchronized neuronal firing in primate somatosensory cortex. Nature 404:187-190.

Tiesinga PH, Fellous JM, Jose JV, Sejnowski TJ (2001) Computational model of carbachol-induced delta, theta, and gamma oscillations in the hippocampus. Hippocampus 11:251-274

van Burik M, Pfurtscheller G (1999) Functional imaging of postmovement beta event-related synchronization. J Clin Neurophysiol 16:383-390.

Van Veen BD, van Drongelen W, Yuchtman M, Suzuki A (1997) Localization of brain electrical activity via linearly constrained minimum variance spatial filtering. IEEE Trans Biomed Eng 44:867-880.

Worden MS, Foxe JJ, Wang N, Simpson GV (2000) Anticipatory biasing of visuospatial attention indexed by retinotopically specific alpha-band electroencephalography increases over occipital cortex. J Neurosci 20:RC63(1-6).

Zangaladze A, Epstein CM, Grafton ST, Sathian K (1999) Involvement of visual cortex in tactile discrimination of orientation. Nature 401:587-590.

Zhang M, Weisser VD, Stilla R, Prather SC, Sathian K (2004) Multisensory cortical processing of object shape and its relation to mental imagery. Cogn Affect Behav Neurosci 4:251-259. 